\title{
ARTRODESE DO PUNHO COM FIXAÇÃO MÍNIMA PRESERVANDO AS ARTICULAÇÕES CARPOMETACARPIANAS
}

\author{
WRIST ARTHRODESIS WITH MINIMAL INTERNAL FIXATION \\ PRESERVING THE CARPOMETACARPAL JOINTS
}

Arlindo Gomes Pardini Júnior', Rodolfo Fonseca Pádua Gonçalves², Afrânio Donato Freitas ${ }^{3}$, Antonio Barbosa Chaves ${ }^{3}$

\section{RESUMO}

Objetivo: Artrodese do punho é uma cirurgia que deve ser sempre considerada em casos de patologias que alteram sua estrutura anatômica e funcional. Em geral os resultados são muito satisfatórios, principalmente no alívio da dor e na maioria das vezes a melhora funcional é considerável. Existem várias técnicas descritas, com variações no método de fixação interna e a maioria delas incluindo as articulações carpometacarpianas na fusão. O objetivo deste estudo é avaliar os resultados da artrodese do punho com uma técnica mais simples, mais biológica, menos dispendiosa e que não inclui as articulações carpometacarpianas. Métodos: foram avaliados 15 pacientes (seis sequelas de traumatismo, quatro de artrite reumatoide, três de Kienbock grau IV, um de Preiser e um de pan-artrose). A técnica consistiu no uso de placa óssea do ilíaco e fixação com fios de Kirschner, sem incluir as articulações carpometacarpianas. Resultados: A avaliação foi feita conforme o tempo de consolidação (93\% em sete semanas); movimentos dos dedos e de pronossupinação; da força de pinça e preensão; avaliação funcional pelo questionário DASH, da dor e satisfação dos pacientes. Em geral, os resultados foram semelhantes aos das outras técnicas mais agressivas e a não inclusão das articulações carpometacarpianas na artrodese não afetou o resultado final. Conclusão: A artrodese do punho com fixação através de fios de Kirschner e uso de placa óssea do ilíaco preservando as articulações carpometacarpianas dá resultado bom ou excelente, não inferior ao de outras técnicas descritas. Apresenta, porém uma grande vantagem sobre as demais: é menos agressiva, mais barata e não apresenta os inconvenientes do uso de placas e parafusos sujeitos a maiores complicações

Descritores - Punho; Artrodese; Enxerto ósseo; Articulações carpometacarpais; Fixadores internos

\section{ABSTRACT}

Objective: Wrist arthrodesis is a surgical procedure that should always be considered in pathologies where there is alteration of the anatomical and functional structures. In general, the results are very satisfactory, particularly for pain relief, and in the majority of cases, there is considerable functional improvement. Various techniques are described, with different methods of internal fixation, most of which include the carpometacarpal joints (CMJ) in the fusion. The objective of this study is to evaluate the results of wrist arthrodesis through a technique which is simpler, more biological, more inexpensive, and does not involve the carpometacarpal joints. Methods: 15 patients with wrist arthrodesis were evaluated (6 with sequelae of trauma, 4 Rheumatoid Arthritis, 3 Kienbock's grade IV, 1 Preiser and 1 panarthrosis). The technique consisted of the use of an iliac bone plate and internal fixation with Kirschner wires, avoiding the carpometacarpal joints. Results: The evaluation was based on consolidation time (93\% in 7 weeks); movements of the fingers and pronosupination; pinch and grasp strength; functional evaluation through the DASH questionnaire, pain and patient satisfaction. In general, the results were similar to those of other, more aggressive techniques, and the non-inclusion of the carpometacarpal joints did not affect the final result. Conclusion: wrist arthrodesis with fixation using Kirschner wires and the use of an iliac bone plate, preserving the carpometacarpal joints, gives good or excellent results which are similar to those of other techniques described. However it presents major advantages over other methods: it is less aggressive, less expensive, and does not have the inconvenience and complications associated the use of plates and screws.

Keywords - Wrist; Arthrodesis; Bone graft; Carpometacarpal joint; Internal fixators

\footnotetext{
1 - Livre-Docente em Ortopedia e Traumatologia. Chefe do Serviço de Cirurgia da Mão do Hospital Ortopédico de Belo Horizonte.

2 - Ex-Residente de Cirurgia da Mão do Serviço de Cirurgia da Mão do Hospital Ortopédico de Belo Horizonte.

3 - Cirurgião de Mão do Hospital Ortopédico e Hospital Belo Horizonte.
}

Trabalho realizado no Hospital Ortopédico de Belo Horizonte, MG.

Correspondência: Rua Prof. Otávio Coelho de Magalhães, 111. 30210-300 - Belo Horizonte, MG - E-mail: centrodeestudosho@hotmail.com 


\section{INTRODUÇÃO}

Artrodeses, de modo geral, são consideradas cirurgias de salvação. No punho, quando dor, deformidade e instabilidade comprometem a função da mão, sua estabilização através da fusão é um procedimento de reconhecida validade ${ }^{(1-3)}$. Várias técnicas têm sido descritas para as artrodeses radiocarpais, como a simples decorticação com remoção da cartilagem e fixação com fios de Kirschner $^{(4)}$, uso de uma haste de Rush do $3^{0}$ metacarpiano à metáfise do rádio ${ }^{(5,6)}$ deslizamento ou rotação de uma placa óssea da extremidade distal do rádio fixada distalmente nos ossos do carpo ${ }^{(4,5,7)}$, fixação com placa metálica do $3^{0}$ metacarpiano ao radio ${ }^{(8,9)}$, enxerto e fixação com placa óssea modelada da crista ilíaca $^{(10)}$.

A maioria das técnicas recomenda incluir as articulações carpometacarpianas do indicador e/ou do dedo médio na fusão. A finalidade deste trabalho é apresentar a técnica e os resultados da artrodese do punho sem incluir as articulações carpometacarpianas, e sem usar placas metálicas.

Este estudo recebeu a aprovação do Conselho de Ética da instituição.

\section{MÉTODOS}

Foram revistos os prontuários de 15 pacientes submetidos à artrodese do punho (15 punhos) no período de setembro de 1996 a novembro de 2006. Em todos a indicação foi devida à dor e deformidade associadas a grande disfunção da mão, devido a sequelas de traumatismos como a de fratura (cinco casos) e lesão ligamentar crônica (um caso); doença de Kienbock com pan-artrose (três casos), artrite reumatoide e psoriática (quatro casos), sequela de doença de Preiser (um caso) e pan-artrose de causa indeterminada (um caso).

Oito pacientes eram homens e sete mulheres; idade variou de 18 a 62 anos, com média de 44,5 anos. O lado dominante predominou em $80 \%$ dos pacientes. O seguimento médio foi de 66,2 meses, variando de 15 a 136 meses.

\section{Técnica cirúrgica}

A via de acesso é através de uma incisão dorsal em “S”, centrada no tubérculo de Lister e cerca de $3 \mathrm{~cm}$ proximal e distal a ele (Figura 1A). Preservar cuidadosamente os ramos sensitivos dos nervos radial e ulnar e as veias que correm longitudinalmente. $\mathrm{O}$ tendão do longo extensor do polegar é removido de trás do tubérculo de Lister e afastado radialmente juntamente com os tendões dos exten- sores radiais do carpo. O quarto compartimento extensor é afastado ulnarmente, expondo a cápsula articular. Esta é aberta por uma incisão em "H”, cujo ramo central horizontal se localiza na articulação radiocarpal (Figura 1B). Os dois ramos longitudinais de cerca de $2 \mathrm{~cm}$ de largura são incisados até o osso e com um osteótomo levantamse dois retalhos osteocapsulares de base proximal e distal (Figura 1C ). O retalho distal inclui a cortical dorsal do semilunar, do escafoide e do capitato, preservando as articulações carpometacarpianas e o retalho proximal inclui cerca de $2 \mathrm{~cm}$ do dorso da extremidade distal do rádio. Fletindo-se o punho faz-se a decorticação, removendo-se toda a cartilagem radiocarpal e escafo-semilunar-capitato. Retira-se da parede lateral do ilíaco uma tábua óssea de cerca de 3,5cm X 2,0 cm. A parede lateral desse osso tem uma curvatura de aproximadamente $15^{\circ}$ e a placa óssea, adaptada ao leito preparado no dorso do punho, dará a posição desejada para a artrodese $\left(15^{\circ}\right.$ a $20^{\circ}$ de extensão) (Figuras 2A e B). As superfícies cruentas das articulações radiocárpica e intercarpais são preenchidas com osso esponjoso da crista ilíaca. A tábua óssea é fixada ao leito com dois fios de Kirschner de 1,0mm e o punho fixado com dois fios cruzados de $1,5 \mathrm{~mm}$ na posição desejada. Os fios são cortados para que fiquem cerca de 5 a $6 \mathrm{~mm}$ por fora da pele. Os retalhos osteocapsulares são suturados sobre a placa óssea, obtendo-se assim um leito regular para o deslizamento dos tendões extensores. A retinácula e a pele são suturadas da maneira usual. Em nenhum caso foi feita a remoção da extremidade distal da ulna.

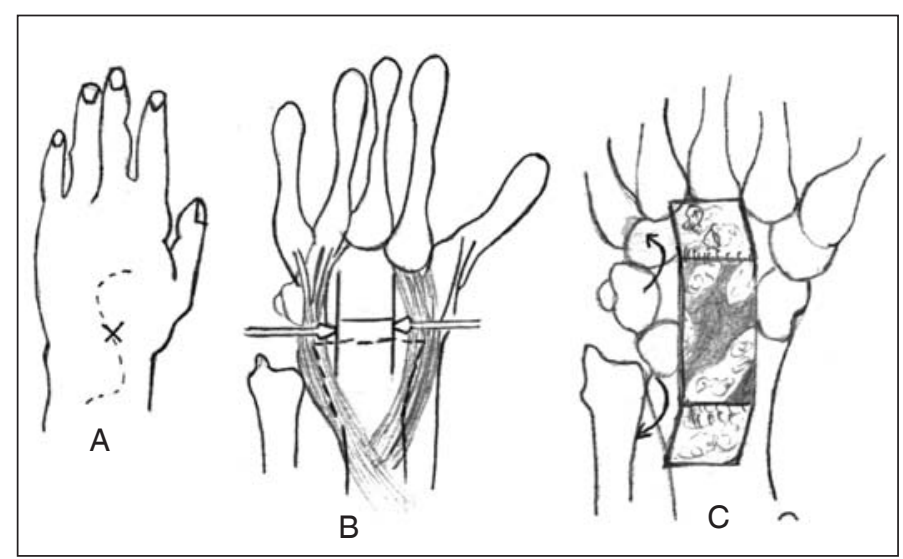

Figura 1 - A) Via de acesso para a artrodese do punho; incisão em "S" centrada no tubérculo de Lister. B) Os tendões extensores dos dedos do $4 .^{\circ}$ compartimento são afastados ulnarmente e os extensores radiais e o extensor longo do polegar, radialmente. A cápsula é aberta em "H" com o ramo central na articulação radiocarpiana. C) Retalhos osteocapsulares levantados. Fazendo-se a flexão do punho, decorticam-se as superfícies articulares radiocarpais e escafo-semilunar-capitato, preservando as articulações carpometacarpianas 

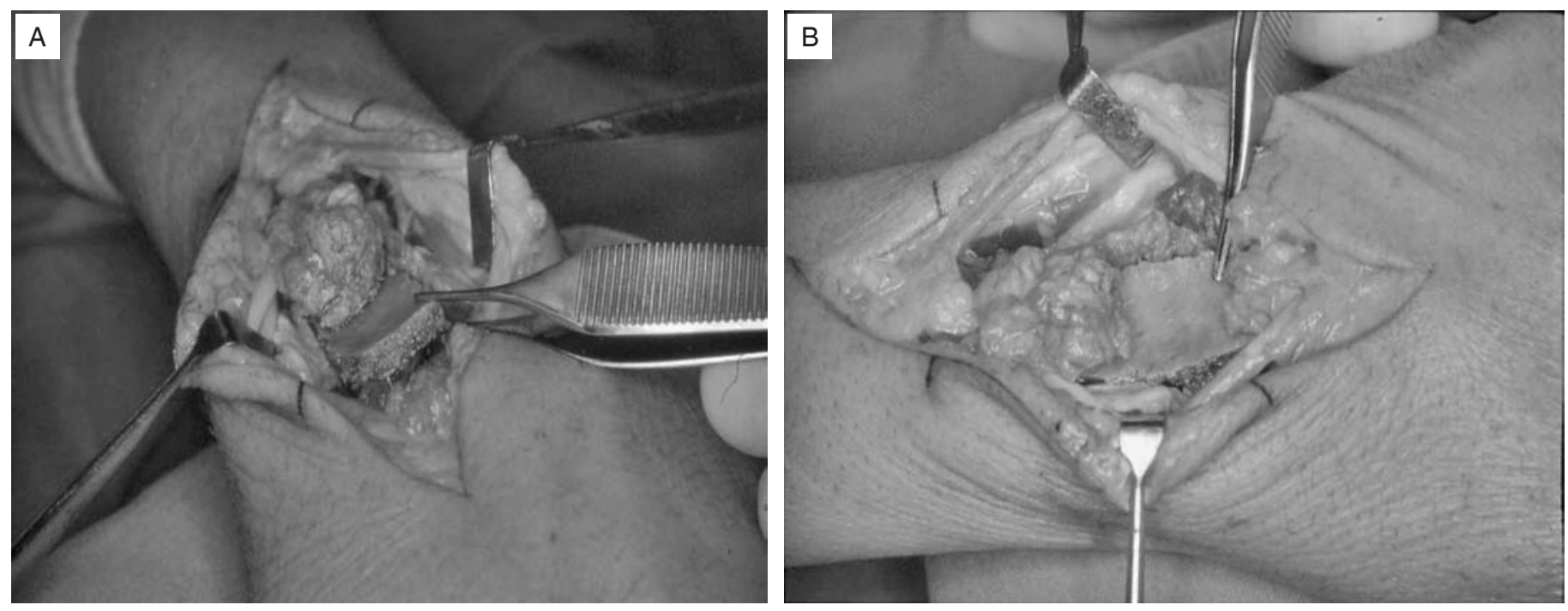

Figura 2 - A) Com o punho fletido, a placa óssea do ilíaco é encaixada sob os retalhos osteocapsulares proximal (radio) e distal (semilunar, escafoide, capitato). B) Ao se fazer a extensão do punho a $15^{\circ-2} 0^{\circ}$, a tábua óssea se adapta no leito preparado. Os retalhos são suturados sobre a placa óssea e o punho é fixado com dois fios de Kirschner cruzados; a placa óssea é fixada com dois fios mais finos e divergentes

Coloca-se um gesso longo, axilopalmar, que deve ser fendido para evitar edema acentuado dos dedos no pósoperatório. Após quatro semanas o gesso longo é trocado por um curto, faz-se controle radiográfico e os fios de Kirschner são removidos. O gesso curto é removido após três semanas e feito novo controle radiográfico. Caso ainda não haja evidência de consolidação, colocase uma tala gessada por mais três semanas.

A análise dos resultados foi baseada na revisão dos prontuários e nas avaliações objetiva e subjetiva. A avaliação objetiva foi através de radiografias convencionais em duas incidências, dos movimentos de pronação e supinação, flexo-extensão dos dedos e da força de pinça e de preensão. A análise subjetiva foi feita através do questionário DASH (Disability of the Arm, Shoulder and Hand $)^{(11)}$, da avaliação da dor pela escala analógicovisual e da satisfação do paciente.

Dos 15 pacientes, 10 consentiram em participar deste estudo. Destes, sete compareceram pessoalmente e três, devido à impossibilidade de se deslocar até o hospital, foram avaliados através dos questionários e respostas via e-mail e/ou telefone.

A análise estatística foi realizada pelo programa EpiInfo através do qual foram calculadas as médias e os desvios-padrão.

\section{RESULTADOS}

Dos 15 pacientes cujos prontuários foram analisados, foi obtida consolidação em 14 punhos (93,3\%) em um tempo médio de sete semanas (Figura 3). O único paciente em que não foi obtida consolidação foi reoperado e a pseudartrose radiocárpica se consolidou após enxerto ósseo. O resultado quanto aos movimentos de antebraço e dedos foi muito satisfatório, obtendo-se a média de $64^{\circ}$ para a pronação e $56^{\circ}$ para a supinação. Em todos os pacientes a flexo-extensão dos dedos era normal.

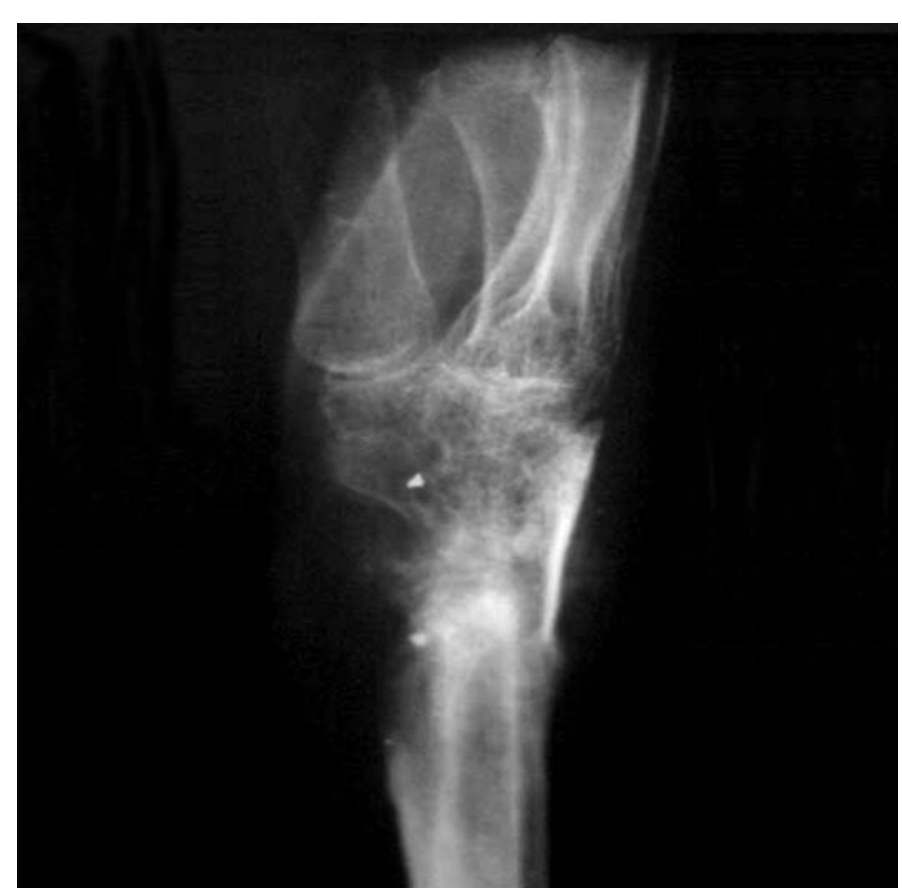

Figura 3 - Radiografia em perfil do punho artrodesado com fixação mínima. Observar a placa óssea dorsal e as articulações carpometacarpianas livres. Os fios de Kirschner que fixavam a placa óssea e a articulação radiocarpal foram removidos 
Quanto à força de preensão medida com o dinamômetro Jamar foi em média de $25,616 \mathrm{~kg} / \mathrm{f}$, com valores entre 18,66 e 32,33 (desvio- padrão (DP) = 5,393) ou $96,3 \%$ com relação ao lado não operado. A força da pinça de polpa digital medida com um pinchmeter foi em média de14,281kg/f, variando entre 12,66 e $15,00 \mathrm{~kg} / \mathrm{f}$ (DP $=0,757)$ ou $94,6 \%$ do lado oposto. Fato interessante foi que a força da pinça lateral foi superior em média à do lado não operado (lado operado $15,189 \mathrm{~kg} / \mathrm{f} ; \mathrm{DP}=0,857$ e o não operado $14,234 \mathrm{~kg} / \mathrm{f}$; $\mathrm{DP}=1,642)$.

Na avaliação subjetiva empregamos o questionário DASH. Este teste avalia os sintomas e a função, isto é, a capacidade do paciente de realizar certas atividades através de 30 questões, como escrever, girar uma chave, usar talheres, fraqueza, etc. Pelo menos 27 desses itens devem ser respondidos. Cada questão tem cinco respostas com valor de 1 a 5 , conforme a dificuldade: nenhuma, pouca, moderada, severa e incapacidade. O resultado é submetido a uma fórmula matemática para transformá-la em múltiplo de $100^{(11)}$. Quanto mais alto o escore, pior a incapacidade e vice-versa. A média de pontuação do questionário DASH foi de 27,004 (DP = 15,717), variando de 0 a 45 (em 100).

Na avaliação da dor através da escala analógica visual, todos os pacientes indicaram a extremidade do lado esquerdo da escala, ou seja, ausência de dor.

Quanto à satisfação com o resultado cirúrgico, todos os pacientes se declararam satisfeitos, sendo que dos 10 avaliados pessoalmente, oito retornaram às atividades anteriores e apenas dois funcionários públicos foram aposentados pela Previdência Social, embora seus resultados pelo DASH tenham sido bons e terem declarado estar satisfeitos com a cirurgia.

\section{DISCUSSÃO}

Artrodese do punho é um procedimento de salvação que acarreta limitações aos pacientes, mas tem um importante papel como meio de alívio da dor e, quando bem indicada e realizada, pode resultar em boa função e melhora da qualidade de vida ${ }^{(12-14)}$.

A técnica por nós empregada tem vantagens indiscutíveis sobre a que usa placa metálica para fixar a artrodese e descrita pelo grupo $\mathrm{AO}^{(15)}$. A fixação mínima com fios de Kirschner mostrou-se eficaz, apresentando consolidação em mais de 93\% dos casos, semelhante à de outras técnicas descritas ${ }^{(6,16)}$. A fixação com placa metálica, principalmente em pacientes reumatoides com intensa osteoporose, pode ser problemática devido ao risco de soltura dos parafusos. Além disso, é uma cirurgia mais agressiva e necessita um descolamento maior de partes moles. Em pacientes magros, a saliência da placa sob a pele, principalmente quando fixada no metacarpiano, pode causar transtorno aos pacientes. Segundo Urbaniak ${ }^{(9)}$, a desvantagem do uso da placa metálica é a necessidade de exposição mais extensa e a frequente necessidade de remover a placa após a consolidação devido à sua proeminência subcutânea ou ao atrito nos tendões extensores. Zachary e Stern ${ }^{(17)}$, em 73 artrodeses em que usaram placa metálica (AO), tiveram 82 complicações em 50 punhos, sendo 40 em curto prazo (relacionados com a ferida cirúrgica, falta de extensão completa dos dedos, déficit neurológico transitório, infecção e dor na articulação radioulnar distal) e 42 em longo prazo, e destas a placa foi removida em 14 casos. Essas incluíram: proeminência dolorosa da placa, afrouxamento dos parafusos, fratura do $3 .^{\circ}$ metacarpiano e do rádio e rigidez metacarpofalangiana.

Acreditamos que nos casos em que não estão afetadas, as articulações carpometacarpianas (CM) não necessitam ser incluídas na artrodese do punho. Observamos em alguns casos que, após a fusão, ocorre aumento na amplitude de movimentos dessas articulações, fato já notado por Urbaniak ${ }^{(9)}$. Abbott et al ${ }^{(18)}$ já haviam demonstrado a importância dos movimentos carpometacarpianos durante a preensão.

Nagy e Buchler ${ }^{(19)}$ fizeram um estudo comparativo entre 47 artrodeses de punho com artrodese carpometacarpiana e 34 em que essa articulação não foi incluída na fusão. Em todos os casos usaram placa metálica fixada no $3^{\circ}$ metacarpiano (nos casos sem fusão CM a placa fazia apenas uma ponte sobre essa articulação). No $1^{\circ}$ grupo, dos 47 casos, 20 evoluíram com pseudartrose, sendo que, destes, 11 eram dolorosas e necessitaram cirurgia adicional. No $2^{\circ}$ grupo, dos 34 casos, apenas um evoluiu com dor.

Em nenhum dos nossos pacientes houve necessidade de remoção da extremidade distal da ulna. Isso não afetou o resultado funcional com relação aos movimentos de pronação e supinação, e, embora com algum déficit, não houve prejuízo para as atividades da vida diária dos pacientes. Esses achados foram semelhantes aos relatados por Barbieri et $a^{(14)}$.

A força de preensão da mão operada comparada com a da não operada foi maior em nossos casos (96\%) do 
que em outros autores ${ }^{(14,20,21)}$. A força da pinça digital de ponta (polpa-polpa) foi em média semelhante à de outras técnicas, porém, a pinça lateral foi surpreendentemente maior que a do lado não operado.

O resultado do questionário DASH, um dos métodos de avaliação funcional da mão mais utilizado atualmente, apresentou pontuação média de 27 (em que 0 = sem incapacidade, 100 = totalmente incapaz) semelhante aos resultados de Adey et al $^{(21)}$ e pouco melhor que o de Wieloch et al $^{(22)}(35,5)$ e bem melhor que o alcançado por De Smet e Truyen ${ }^{(23)}$ (que foi de 44). Esses autores usaram duas técnicas diferentes e não diferenciaram os resultados conforme o procedimento empregado. Nossos resultados foram também superiores aos de Sauerbier et al ${ }^{(24)}$, que obtiveram média de 51 pontos em seus pacientes artrodesados que eram portadores de artrose radiocarpal ou doença de Kienbock.

Com relação à dor, o resultado coincidiu com os de Barbieri et al $^{(14)}$ isto é, só se manifestava com atividades que exigiam pronossupinação mais vigorosa. Todos os pacientes avaliados se disseram satisfeitos com o resultado da cirurgia e se submeteriam novamente a esse tratamento.

\section{CONCLUSÃO}

A artrodese do punho, com fixação mínima, isto é, com fios de Kirschner, é menos agressiva, de baixo custo e tecnicamente simples. A remoção dos fios não requer procedimentos maiores, o material biológico é autógeno e o índice de complicações é mínimo. Pela nossa experiência, não há necessidade de inclusão das articulações carpometacarpianas na artrodese e, em geral, os pacientes se mostram satisfeitos com o resultado desta cirurgia.

\section{AGRADECIMENTOS}

Os autores agradecem ao Dr. Saulo Garzedim Freire pela avaliação estatística deste trabalho.

\section{REFERÊNCIAS}

1. Louis DS, Hankim FM. Arthrodesis of the wrist: past and present. J Hand Surg Am. 1986;11(6):787-9.

2. Rayan GM. Wrist arthrodesis. J Hand Surg Am. 1986;11(3):356-64.

3. Pardini AG, Chaves AB. Artrodeses na mão e no punho. In: Pardini A, Freitas A. Cirurgia da mão - Lesões não traumáticas. Rio de Janeiro: MedBook; 2008. p.595-610.

4. Flat AE. The care of the rheumatoid hand. 2nd ed. St. Louis: Mosby; 1968.

5. Mannerfelt L, Malmsten M. Arthrodesis of the wrist in rheumatoid arthritis. A technique without external fixation. Scand J Plast Reconstr Surg. 1971;5(2):124-30

6. Rauhaniemi J, Tiusanen H, Sipola E. Total wrist fusion: a study of 115 patients. J Hand Surg Br. 2005;30(2):217-9.

7. Stein I. Gill turnabout radial graft for wrist arthrodesis. Surg Gynecol Obstet. 1958;106(2):231-6.

8. Barbieri CH, La Banca Junior J, Sakashita AF. Artrodese do punho com fixação interna rígida. Rev Bras Ortop. 1987;22(6):155-62.

9. Urbaniak JR. Arthrodesis of the hand and wrist. In: Evarts CM. Surgery of the musculoskeletal system. 2nd ed. New York: Churchill Livingstone; 1990. p.767-86.

10. Haddad RJ, Riordan DC. Arthrodesis of the wrist. A surgical technique. J Bone Joint Surg Am. 1967;49(5):950-4

11. Solway S, Beaton DE, McConnell S, Bombardier C. The DASH outcome measure user's manual. 2nd ed. Toronto: Institute for Work and Health; 2002.

12. Van Gemert JG. Arthrodesis of the wrist: a clinical radiographic and ergonomic study of 66 cases. Acta Orthop Scand Suppl. 1984;210:1-146.

13. De Smet L. Does restricted wrist motion influence the disability of the upper limb? Acta Orthop Belg. 2007;73(4):446-50.
14. Barbieri $\mathrm{CH}$, Mazzer N, Elui VMC, Fonseca MCR. Resultados funcionais da artrodese do punho. Acta Ortop Bras. 2002;10(1):17-24.

15. Heim U, Pfeiffer KM. Small fragment set manual: technique recommended by the ASIF group. 2nd ed. New York: Springer Verlag; 1982. p. 53-54, 133-4, 143, 160-1.

16. Barbieri $\mathrm{CH}$, Mazzer N, Kfuri Júnior M, Nishimura MT, Elui VC. Artrodese do punho com fixação interna rígida: avaliação funcional. Rev Bras Ortop. 1994;29(6):411-5.

17. Zachary SV, Stern PJ. Complications following AO/ASIF wrist arthrodesis. J Hand Surg Am. 1995;20(2):339-44.

18. Abbott LC, Saunders JBBDM, Bost FC. Arthrodesis of the wrist with the use of grafts of cancellous bone. J Bone Joint Surg. 1942; 24:883-98.

19. Nagy L, Buchler U. AO wrist arthrodesis: with and without arthrodesis of the third carpometacarpal joint. J Hand Surg Am. 2002;27(6):940-7.

20. Toma CD, Machacek, P, Bitzan P, Assadian O, Trieb K, Wanivenhaus A. Fusion of the wrist in rheumatoid arthritis: a clinical and functional evaluation of two surgical techniques. J Bone Joint Surg Br. 2007;89(12):1620-6.

21. Adey L, Ring D, Jupiter JB. Health status after total wrist arthrodesis for posttraumatic arthritis. J Hand Surg Am. 2005; 30(5):932-6.

22. Wieloch $\mathrm{PT}$, Martini AK, Jung M, Daecke W. Long-term results after mediocarpal and total arthrodesis of the wrist: a matched paired study. Orthop Ihre Grenzgeb. 2006;144(2):206-11.

23. De Smet L, Truyen J. Arthrodesis of the wrist for osteoarthritis: outcome with a minimum follow-up of 4 years. J Hand Surg Br. 2003;28(6):575-7.

24. Sauerbier M. Klug S, Bickert B, Germann G. Subjective and objective outcomes after total wrist arthrodesis in patients with radiocarpal arthrosis or Kienbock's disease. Chir Main. 2000;19(4):223-31. 